\title{
Apollo 11 Reloaded: Optimization-based Trajectory Reconstruction
}

\author{
Marco Sagliano* \\ German Aerospace Center / Japan Aerospace Exploration Agency, Germany / Japan
}

\begin{abstract}
This paper wants to be a tribute to the Apollo 11 mission, that celebrated its $5^{\text {th }}$ anniversary in 2019. By using modern methods based on numerical optimization we reconstruct critical phases of the original mission, and more specifically the ascent of the Saturn V, the translunar injection maneuver that allowed the crew to leave the Earth's sphere of influence, and the Moon landing sequence, starting from the powered descent initiation. Results were computed by employing pseudospectral methods, and show good agreement with the original post-flight reports released by NASA after the successful completion of the mission.
\end{abstract}

\section{Introduction}

July, 20, 1969 marked an historical achievement in the humankind history. For the first time two men walked on a celestial body that was not the Earth, fixing a fundamental milestone in the human exploration history. This success was the culmination of a gigantic effort, both from the technical and the economic point of view, made by the United States to respond to the earlier successes of the Soviet space program, first with the creation and the successful launch of the first artificial satellite, the Sputnik in 1957 [1, 2], and later with the first man able to reach the space and journey into outer space with its Vostok 1, Yuri Gagarin, in 1961 [3]. These were the first formal steps of what went down in history as the space race [4] in general, and the Moon race in particular [5].

Despite the initial technological gap the American progress in space gained momentum over the years, and the setup of the Apollo missions [6] represented the highest point of success of the entire US space program. To be able to achieve such a goal several new technologies needed to be developed. Among these there was certainly the capability to compute trajectories able to satisfy all the requirements all along the mission. This despite the strict requirements in terms of available computational power of the Apollo Guidance Computer [7] and the Launch Vehicle Digital Computer used to guide the Saturn V [8]. For the ascent guidance the rocket employed the so-called iterative-path adaptive guidance, that exploited optimal control theory [9], and a modified version of the tangent linear steering law, where its parameters were constantly updated during the flight. Special care was taken during the last seconds before the engine cut-off to avoid a singularity in the solution.

Another fundamental phase was represented by the Translunar injection (TLI) maneuver, that allowed the spacecraft to leave the Earth's sphere of influence to reach the Moon. For Apollo 11 the maneuver was conceived to place the Columbus module on a free-return path [10], and this choice required accurate attitude and position conditions to be met at the end of the maneuver. The third, and most important phase was the Moon landing: given the aforementioned computational limitations, NASA engineers compensated for it in terms of commitment, creativity and know-how. A brilliant example of this attitude is the Moon landing guidance, based on the polynomial scheme, which results to be extremely efficient despite its low computational complexity [11], and proven to be also mathematically optimal in its E-guidance form [12].

However, the progress made in the last decades both in terms of computational power and development of refined optimization algorithms enormously extended the plethora of methods and tools available today to analyze the same problems. In this context we can place numerical optimization in general [13], and direct methods [14] in particular. Among the direct methods employed to solve optimal control problems pseudospectral methods occupy a relevant place. These methods [15], based on a non-uniform distribution of the time-steps used to transcribe the problem proved to be very effective for a large class of optimal-control problems [16], including the zero-propellant re-orientation of the International Space Station [17]. Further applications involved atmospheric entry guidance [18, 19], Mars descent and asteroid landing trajectory computation [20], Moon landing reachability analysis [21], attitude stabilization of satellites on elliptical orbits [22] and aircraft trajectory generation problems [23].

In this paper we want to reconstruct three critical phases of the Apollo 11 mission by employing SPARTAN $[19,24,25]$ a tool developed by the German Aerospace Center implementing multi-phase pseudospectral methods based

\footnotetext{
*Visiting GNC Specialist, 7-44-1, Jindaiji Higashimachi, Chofu, Japan, 182-8522
} 
on flipped Legendre-Gauss-Radau polynomials. For a more thorough description we suggest the reader to refer to [26], while in this context we will focus on the modeling of the phases of Apollo 11 mission, based on a critical comparison and integration of the different sources provided by NASA over the years. More specifically, we will not focus on the full trajectory covering the entire mission, but concentrated our efforts on the ascent phase, the TLI maneuver, and the Moon landing phase. Where possible we will highlight the differences in modeling with respect to the original assumptions made by NASA engineers, and provide a comparison of results.

This paper is organized as follows: in Sec. II we will provide a description of the equations and the assumptions required to model the Saturn-V ascent. In Sec. III the TLI maneuver will be described, and the corresponding results documented. Section IV will illustrate the optimal control problem built to compute the Moon landing trajectory. Finally, some conclusions on this work and the future outlook will be drawn in Sec. V.

\section{Ascent}

The entire space program could not exist without a launch system able to safely deliver into orbit the crew and the corresponding payload needed to go to the Moon. This need was satisfied by the Saturn V, depicted in Fig. 1, so far the largest rocket ever built able to successfully fly.

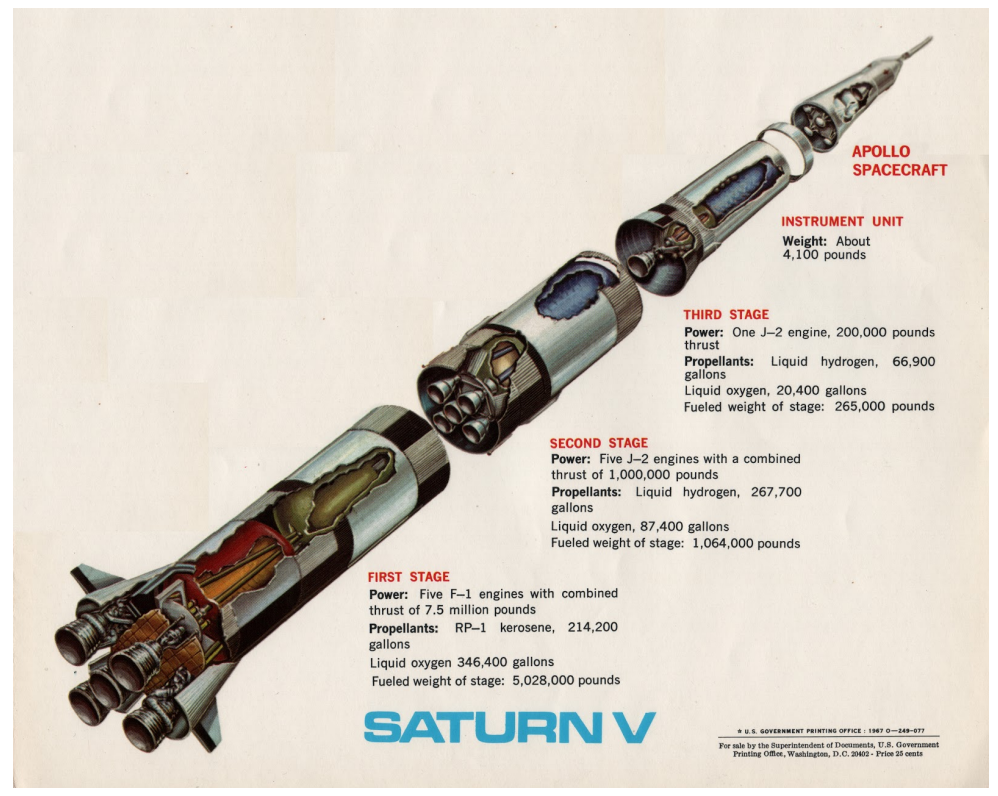

Fig. 1 Saturn V Rocket (courtesy of NASA and U.S. Government).

The rocket consists of 3 stages: the S-IC stage, having 5 F-1 non-throttleable engines, each of them generating 6700 $\mathrm{kN}$ for a total of more than $33 \mathrm{MN}$, the S-II stage, with $5 \mathrm{~J}-2$ engines, each generating $1 \mathrm{MN}$, and the S-IVB stage, equipped with another $\mathrm{J}-2$ engine. In this subsection we will focus on the ascent part, meant as the segment of mission going from the launch to the parking orbit injection, occurring at about $T=+710 \mathrm{~s}$. By looking at the original NASA documentation [27] it was decided to model the problem with five different phases. In the first phase the $5 \mathrm{~F}-1$ engines provide a constant thrust for about $135 \mathrm{~s}$, when an altitude of $44 \mathrm{~km}$ is reached. At this point the central engine is shut down, and the ascent continues with the 4 lateral engines up to an altitude of $68 \mathrm{~km}$, reached at $\mathrm{t}=162 \mathrm{~s}$. When the first-stage separation is performed the rocket continues to increase its speed, sustained by the thrust of the $5 \mathrm{~J}-2$ engines of the S-II stage up to an altitude of $180 \mathrm{~km}$ and a speed of $5.3 \mathrm{~km} / \mathrm{s}$. After $460 \mathrm{~s}$ a MECO command for the central J-2 engine is issued, and, as in the previous phase, the lateral 4 engines continue to provide thrust until a time of about $550 \mathrm{~s}$. Finally, the second stage-separation event occurs, and the S-IVB stage continues its flight with the thrust generated by its only J-2 engine until reaching the parking orbit conditions, after approximately $710 \mathrm{~s}$.

A relevant effort was devoted to the modeling by looking at different sources, including the aforementioned report [27], the entire mission post-flight report [28], the website of Braeunig [29], and the official book containing data and statistics for the entire Apollo program [30]. One of the most important factors is the engine modeling: note that quantities like the thrust, the massflow, and the specific impulse are modeled as look-up table based on the data plotted 
in [27], Figs. 5-3, 6-3, while the drag coefficient as function of the Mach number was obtained by looking at the reconstruction work performed by Braeunig [29]. Examples of these look-up tables for modeling the drag coefficient $C_{D}$ and the massflow for the S-IC and S-II stages are reconstructed in Figs. 2 and 3.

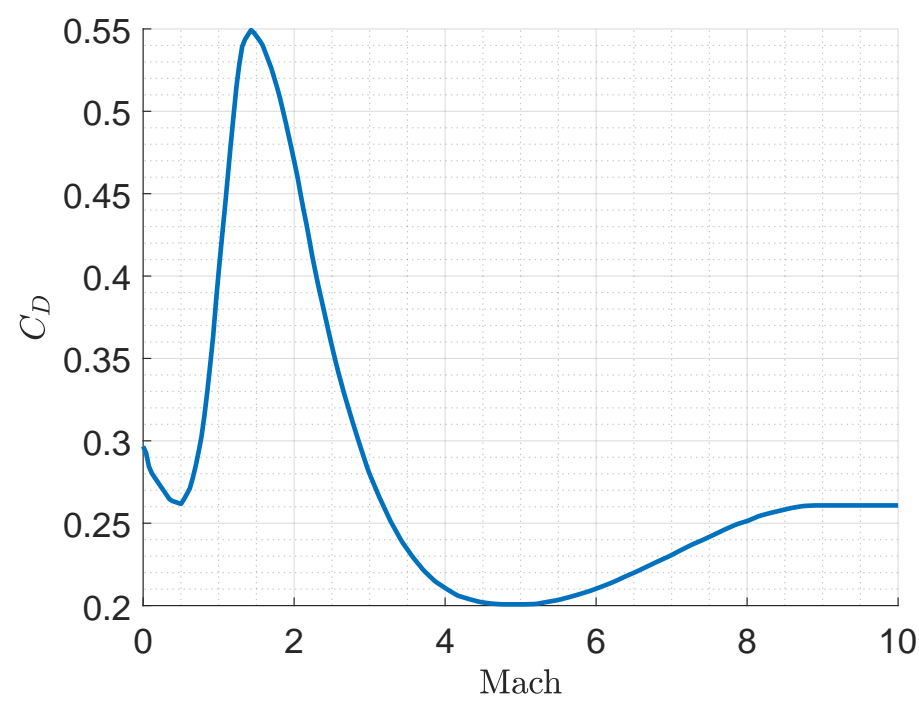

Fig. 2 Saturn V - Estimate of $C_{D}$ based on the work of Braeuenig [29].
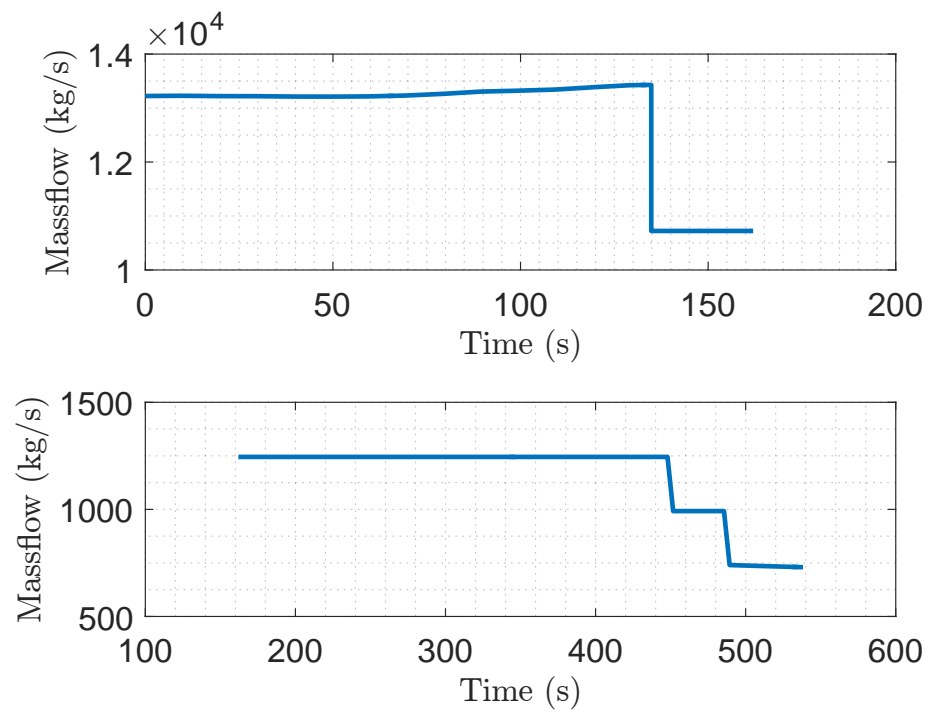

Fig. 3 Saturn V - Reconstruction of massflow for the S-IC stage (top) and the S-II stage (bottom) based on [27], Figs 5-3, 6-3

Finally, for the atmosphere, although not available at the time of the Apollo 11 mission, a US-76 model has been adopted. [31].

The equations of motion used to describe the problem are formulated with respect to the Earth-Centered, Earth-Fixed reference frame. Although more choices are available (e.g., inertial-based representations), this formulation was adopted to make an easier comparison with the original documentation, which contains the trajectory in tabular data in this reference frame ([28], pages C-26-C-37). Note that the fuel consumption cannot be optimized, due to the non-throttleable engines, and the look-up tables model the thrust and the massflow too. Therefore the cost function adopted was aiming at the minimization of the control rate effort. For the sake of easiness of implementation we neglected some more detailed 
aspects, like the ullage motors and the initial roll maneuver described in [27]. The cost function is therefore defined as

$$
\mathcal{J}=\int_{t_{0}}^{t_{f}}\left(\dot{\theta}(t)^{2}+\dot{\psi}(t)^{2}\right) d t
$$

The equations of motion, expressed in ECEF coordinates, are the following

$$
\begin{cases}\dot{\boldsymbol{r}} & =\boldsymbol{v} \\ \dot{\boldsymbol{v}} & =-\frac{\mu}{\|\boldsymbol{r}\|^{3}} \boldsymbol{r}+\frac{T}{m} \boldsymbol{u}+\frac{\boldsymbol{D}}{m}-2 \boldsymbol{\Omega}_{\oplus} \times \boldsymbol{v}-\boldsymbol{\Omega}_{\oplus} \times \mathbf{\Omega}_{\oplus} \times \boldsymbol{r} \\ \dot{m} & =-\frac{T}{g_{0} I_{s p}}\end{cases}
$$

where the control $\boldsymbol{u}$ is the Cartesian unit vector coming from the proper transformation of pitch and yaw angles, with roll assumed equal to 0 . Specifically, the thrust direction in body coordinate is defined as

$$
\boldsymbol{u}_{B O D Y}=\left[\begin{array}{lll}
1 & 0 & 0
\end{array}\right]^{T}
$$

which is a valid assumption for 3-DOF motion. Given the pitch $\theta$ and yaw $\psi$, we can transform this unit vector into its corresponding body-carried North-East-Down representation as

$$
\boldsymbol{u}_{N E D}=\boldsymbol{R}_{3}\left(-\psi-\psi_{L}\right) \boldsymbol{R}_{2}(-\pi / 2-\theta) \boldsymbol{u}_{B O D Y}
$$

with $\boldsymbol{R}_{i}$ that is the rotation matrix around the axis $i$. The final transformation into ECEF representation is then given by

$$
\boldsymbol{u}=\boldsymbol{R}_{N E D}^{E C E F}(\mathbf{r}) \boldsymbol{u}_{N E D}
$$

where the subscript for the ECEF representation is dropped to simplify the notation, and $\boldsymbol{R}_{N E D}^{E C E F}$ which is the rotation matrix from NED to ECEF, only depending on the position of the rocket in ECEF $\boldsymbol{r}$. In the dynamics represented by Eq. (2) the drag force was included, and is simply computed as

$$
D=-\frac{1}{2} \rho\|v\| S C_{D} v
$$

with $\rho$ displaying the atmospheric density, $v$ the velocity vector in ECEF, $S$ the reference section of the Saturn V, assumed equal to $112 \mathrm{~m}^{2}$ [29], and $C_{D}$ is the drag coefficient depicted in Fig. 2. $\Omega_{\oplus}$ is the Earth's rotation rate, equal to $7.292116 \cdot 10^{-5} \mathrm{rad} / \mathrm{s}$. Finally, the angle $\psi_{L}$ is the launch azimuth angle, equal for the Saturn-V to $72.058 \mathrm{deg}$. Note that the last relationship in Eq. (2) is actively used only in phase 5 (corresponding to the third stage), while for phases 1 to 4 we directly invoke the look-up tables shown in Fig. (3), as a single values for the specific impulse is not able to consistently capture the correct massflow evolution.

The initial and final conditions are derived from [27], and transformed into the corresponding ECEF cartesian conditions as follows.

$$
\begin{aligned}
\boldsymbol{r}\left(t_{0}^{(1)}\right) & =\left[\begin{array}{lll}
916245.39 & -5537048.67 & 3020181.60
\end{array}\right]^{\top} \mathrm{m} \\
\boldsymbol{v}\left(t_{0}^{(1)}\right) & =\left[\begin{array}{lll}
0 & 0 & 0
\end{array}\right]^{\top} \mathrm{m} \\
m\left(t_{0}^{(1)}\right) & =2902328 \mathrm{~kg}
\end{aligned}
$$

and

$$
\begin{aligned}
\boldsymbol{r}\left(t_{F}^{(5)}\right) & =\left[\begin{array}{lll}
3360940.44 & -4410922.63 & 3510251.57
\end{array}\right]^{\top} \mathrm{m} \\
\boldsymbol{v}\left(t_{F}^{(5)}\right) & =\left[\begin{array}{lll}
5825.78 & 4543.92 & 133.38
\end{array}\right]^{\top} \mathrm{m} \\
m\left(t_{F}^{(5)}\right) & =166450 \mathrm{~kg}
\end{aligned}
$$




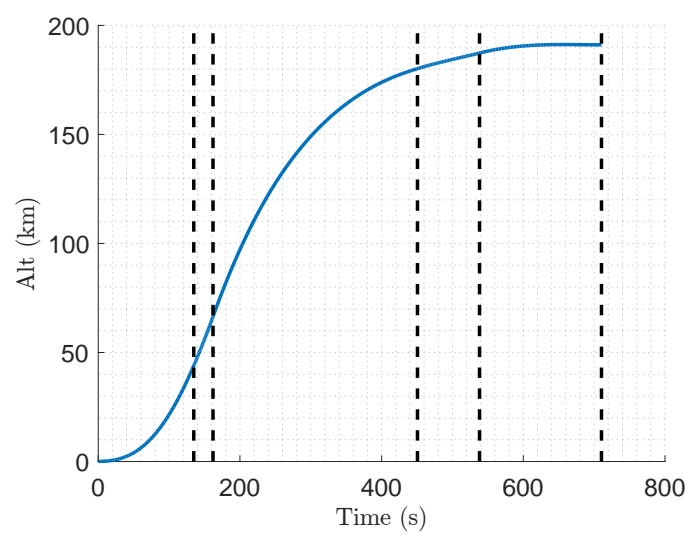

(a) Altitude profile

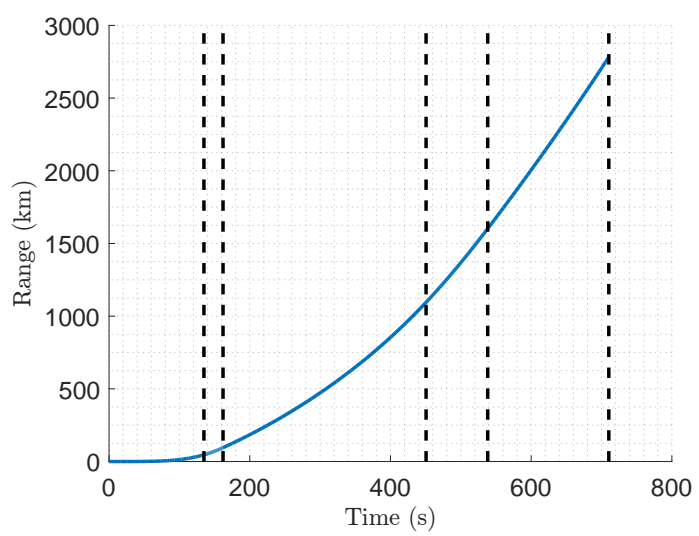

(b) Range profile

Fig. 4 Saturn V ascent: (a) Altitude, and (b) Range.

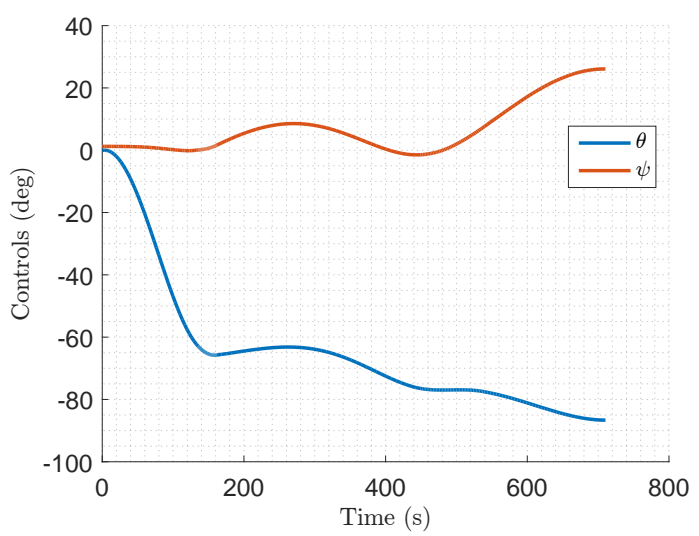

(a) Control profiles

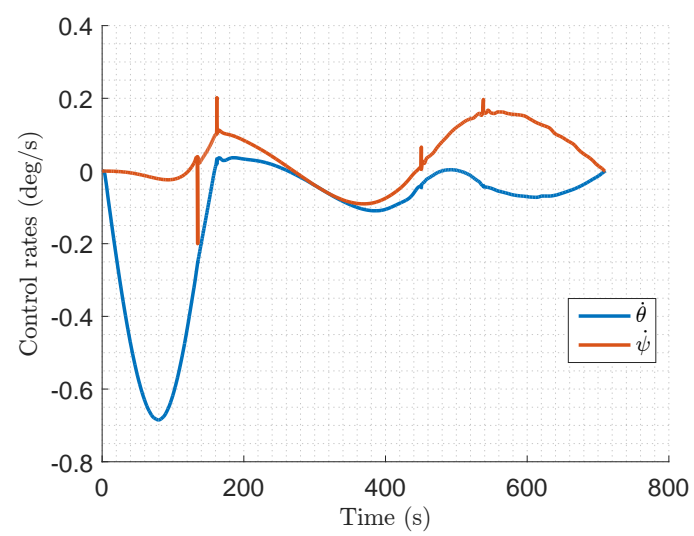

(b) Control rates profiles

Fig. 5 Saturn V ascent: (a) Pitch and yaw, and (b) pitch and yaw rates.

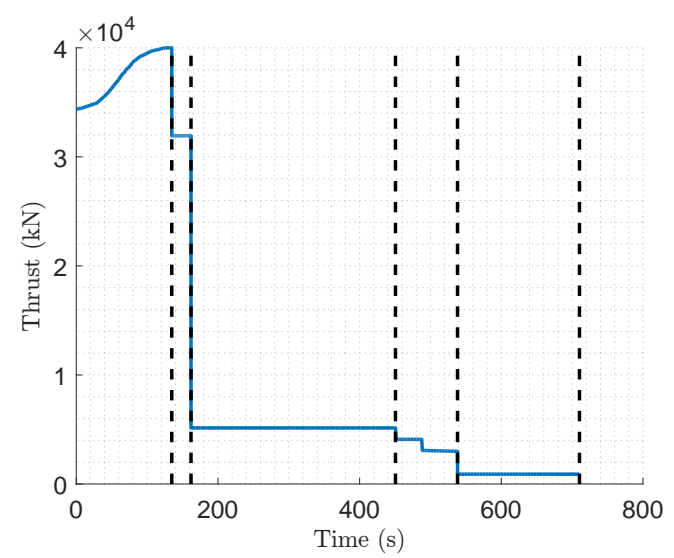

(a) Thrust look-up table

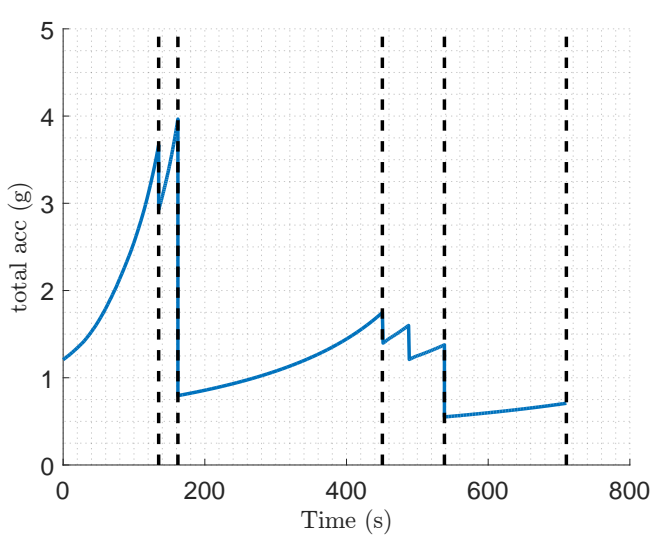

(b) Non-gravitational acceleration

Fig. 6 Saturn $\mathbf{V}$ ascent: (a) Thrust profile, and (b) non-gravitational acceleration expressed in $g$. 


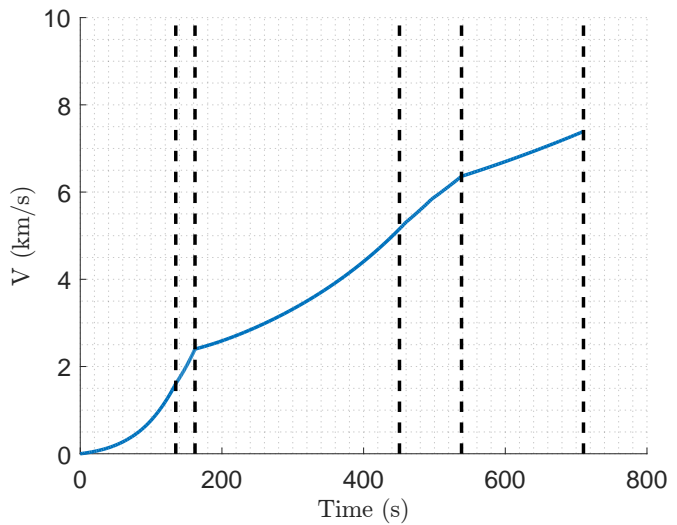

(a) Speed profile

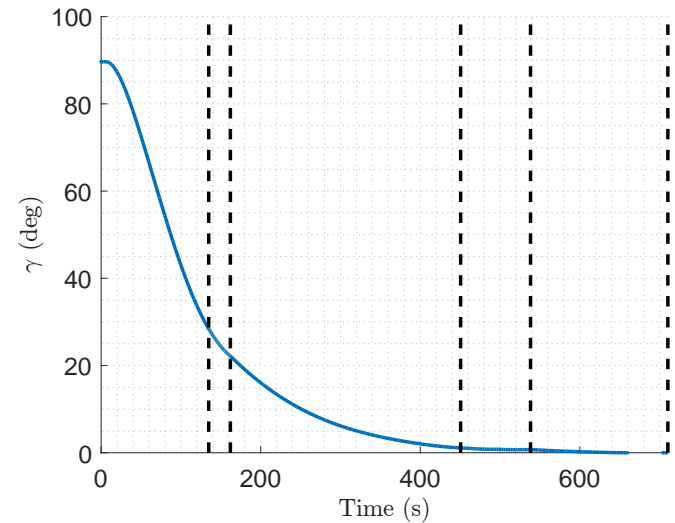

(b) Flight-path angle

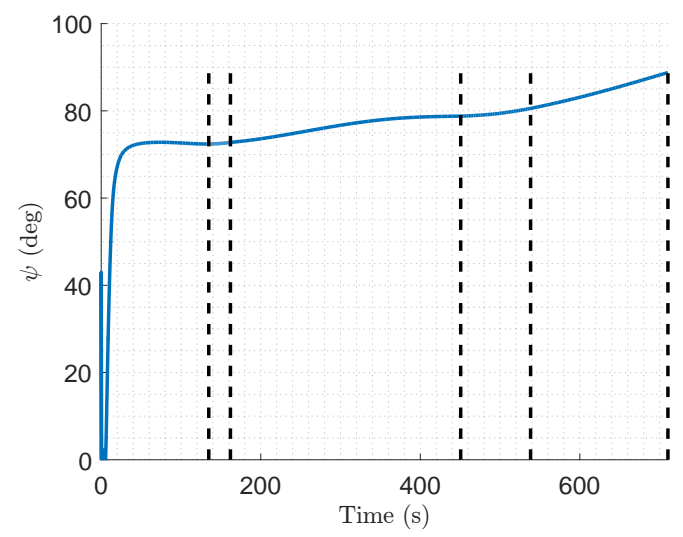

(c) Velocity azimuth angle

Fig. 7 Saturn V ascent: (a) Speed, (b) Flight-path angle, and (c) Velocity-azimuth angle. 


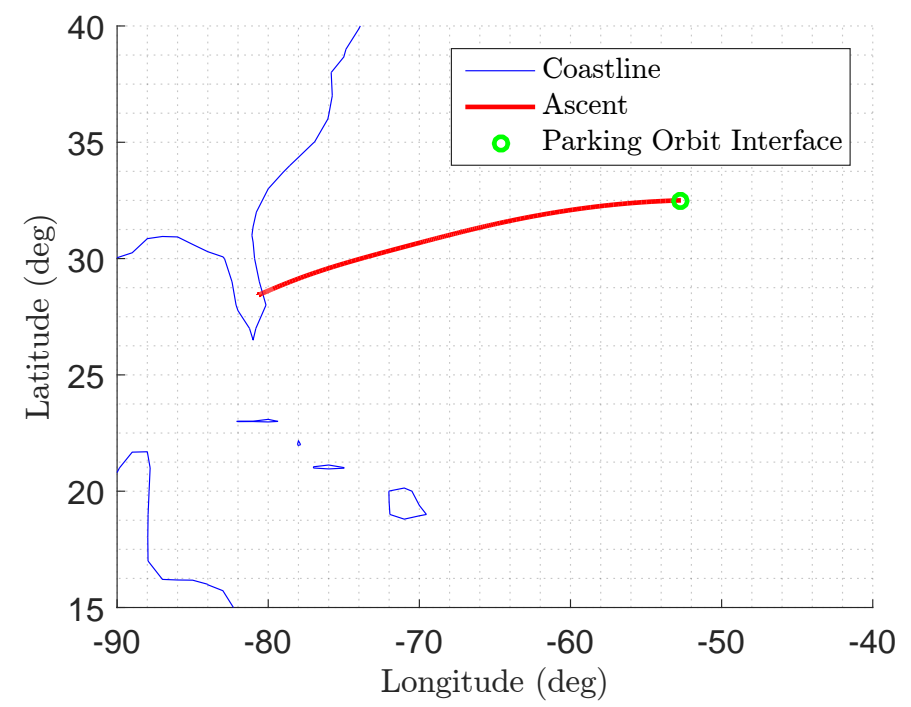

Fig. 8 Saturn V - Groundtrack profile.

with the latter coming from the constraints required to achieve parking orbital injection at $t_{F}=710 \mathrm{~s}$.

Results were obtained with SPARTAN [20, 24, 26], a tool developed by the German Aerospace Center implementing pseudospectral methods, and are depicted in Figs. 4-8.

Figures 4(a) and 4(b) show the altitude and the range flown during the ascent. The black dashed lines identify the several phases of flight. The final altitude and range are fully consistent with the ones described in the original documentation, with the third stage correctly reaching orbital conditions at about $190 \mathrm{~km}$ of altitude after $710 \mathrm{~s}$ of flight. The corresponding controls are depicted in Fig. 5(a). The pitch maneuver resembles quite well the original profile provided by the source ([27], Fig. 10-4). A larger discrepancy is instead observed in the yaw maneuver. The reason for this discrepancy has been identified in the remaining modeling mismatch for what regards mass and thrust, which cause the optimizer to fly slightly more off-plane to meet the final terminal constraints. The control rates are fully satisfied, and in particular is interested to note that the pitch maneuver ends at $t=160 \mathrm{~s}$ without explicitly specifying it in the optimizer, consistently with the original profile, that relies on optimal control theory, and which ends at the same time ([27], Table 2-4).

The thrust profile that was implemented is visible in Fig. 6(a). Note that by using this look-up table we can already implement subtle effects, like the S-II Engine mix-ratio shift, visible at $t=491 \mathrm{~s}$, and described in [27], (Page 6-1, and Fig. 6.3). The non-gravitational acceleration contribution due to the engine is depicted in Fig. 6(b). The profile matches pretty well with the reference data ([27], Fig. 4-3), confirming that all the main events of the ascent were captured with sufficient accuracy.

The validity of the reconstruction is confirmed by the velocity, expressed in spherical coordinates as magnitude, velocity azimuth angle (positive when measured clockwise from North) and flight-path angle. The plots are visible in Fig. 7(a)-7(c). The speed profile is quite accurate with respect to the original data tabulated in [27], and so are the flight-path angle and the velocity azimuth angle. This last variable shows some oscillations during the first seconds. This is not related to the trajectory itself, but is a numerical artifact coming from the fact that this spherical representation is not accurate to represent a motion that is close to be vertical, as its definition becomes ill-conditioned, and singular for perfectly vertical trajectories. This is confirmed by the tabular data in Pages C-27-C-28 of [27], where this phenomenon is also visible from the logged values in there. Finally, the groundtrack is visible in Fig. 8. The trajectory correctly reaches the parking orbit conditions, and is fully consistent with the original figure provided in [28], Fig 3-1. The ascent phase performed by the Saturn-V can be considered complete.

\section{Trans-Lunar Injection}

After the shutdown of the S-IVB occurring at $t=710 \mathrm{~s}$ the vehicle was inserted into a circular orbit. This parking time was spent to check all the subsystems and perform several measurements with the ground stations in preparation 
of the translunar orbit injection (TLI) maneuver, that started at about $t=9856 \mathrm{~s}$. The S-IVB stage together with the spacecraft is depicted in Fig. 9.

This maneuver was fundamental as it allowed the crew to leave the sphere of influence of the Earth, reach the Moon and be placed on a free-return trajectory that could be used in case something went wrong during the following phases of the mission. In this section we focus on this maneuver. The modeling is relatively simpler than the ascent part as no stage separations occur, and therefore one phase is sufficient to capture the dynamic behavior of the spacecraft. As for the previous case we model the equations of motion in ECEF coordinates, to be able to directly compare the results with the tabulated data provided in the AS-506 report [28] (pages C-51-C-62). For the equations of motion and the cost function we can therefore remind to Eqs. (1) and (2). The initial and final conditions are for the TLI defined as follows.

$$
\begin{aligned}
\boldsymbol{r}\left(t_{0}\right) & =\left[\begin{array}{lll}
-6494609 & 849762 & -568593
\end{array}\right]^{\top} \mathrm{m} \\
\boldsymbol{v}\left(t_{0}\right) & =\left[\begin{array}{lll}
-1154 & -6014 & 4137
\end{array}\right]^{\top} \mathrm{m} \\
m\left(t_{0}\right) & =134194 \mathrm{~kg}
\end{aligned}
$$

and

$$
\begin{aligned}
\boldsymbol{r}\left(t_{F}\right) & =\left[\begin{array}{lll}
-6400956 & -1643128 & 1094238
\end{array}\right]^{\top} \mathrm{m} \\
\boldsymbol{v}\left(t_{F}\right) & =\left[\begin{array}{lll}
1807 & -8655 & 5532
\end{array}\right]^{\top} \mathrm{m} \\
m\left(t_{F}\right) & =63196 \mathrm{~kg}
\end{aligned}
$$

Note that while for the first burn of the S-IVB stage the thrust was considered constant, during the second burn this assumption is no longer true, and therefore a specific look-up table was implemented. The thrust profile corresponding to this phase is visible in Fig. 10, and is therefore provided as input to the optimizer. The controls are, as for the ascent part, formulated in terms of pitch and yaw, and their rates, which determine the trajectory leading to the final conditions to be met at $t=10203 \mathrm{~s}$, and which correspond to a correct TLI maneuver. Results are depicted in Figs. 11(a) through 13(c). Given the limited amount of data needed, for easiness of comparison for the reader the tabulated values of position and velocity provided in [28] have directly been imported in Matlab to re-create the plots from the original data, referred to as AS-506.

Figures 11(a) and 11(b) show altitude and range profiles. The final TLI interface is met, and the altitude profile shows very good consistency with the original source data. Control profiles and control rates are depicted in Figs. 12(a) and 12(b). Although not explicitly specified in the documentation, it was assumed an upper bound for control rates equal to $1 \mathrm{deg} / \mathrm{s}$, that was sufficient to perform the maneuver. It is worth mentioning that a direct comparison of the obtained pitch and yaw commands with the original ones provided in the reference is difficult, as no conventions are specified in the original documentation. However, they are considered valid given their envelope, together with the fact that the generated trajectory is consistent with reference sources results. Note that also for this scenario discrepancies can be due also in this case to higher-detail model mismatch (e.g., there is a engine mix-ratio shift commanded during this phase as well that was neglected in this work).

Nevertheless, the overall maneuver resembles quite well the original one, as shown by the speed, flight-path angle and velocity azimuth angle profiles (Figs. 13(a)-13(c)). The maximum difference between the source data and the output of SPARTAN is in the order of $0.01 \mathrm{~km} / \mathrm{s}$ for speed, $0.1-0.2 \mathrm{deg}$ for the flight-path angle, and $0.2-0.4$ deg for the velocity azimuth angle, which can be considered satisfying at this stage. Most important, the final conditions are fully met, which means that, if further propagated this solution will lead the spacecraft towards the Moon, and the mission can continue. 


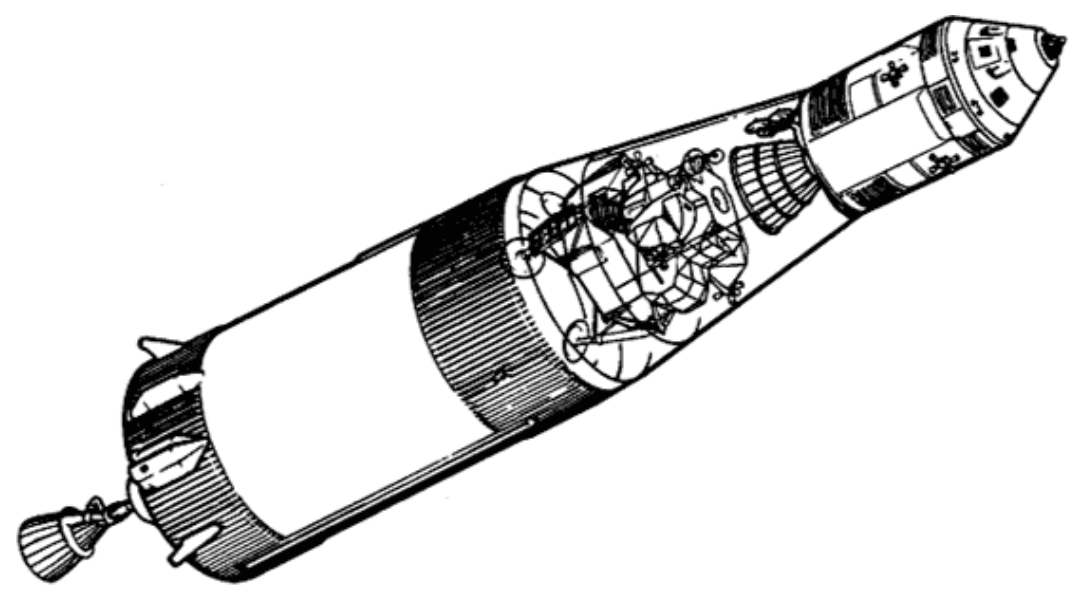

\section{VEHICLE EARTH PARKING ORBIT CONFIGURATION}

(SATURN V THIRD STAGE AND INSTRUMENT UNIT, APOLLO SPACECRAFT)

Fig. 9 S-IVB and payload - configuration for the Translunar Injection Maneuver (courtesy of NASA)

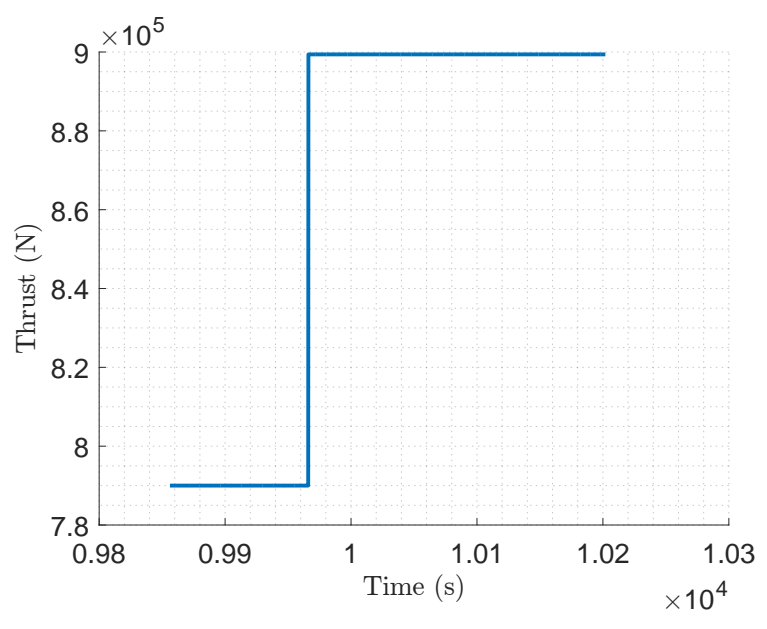

Fig. 10 Thrust profile during the TLI maneuver. 


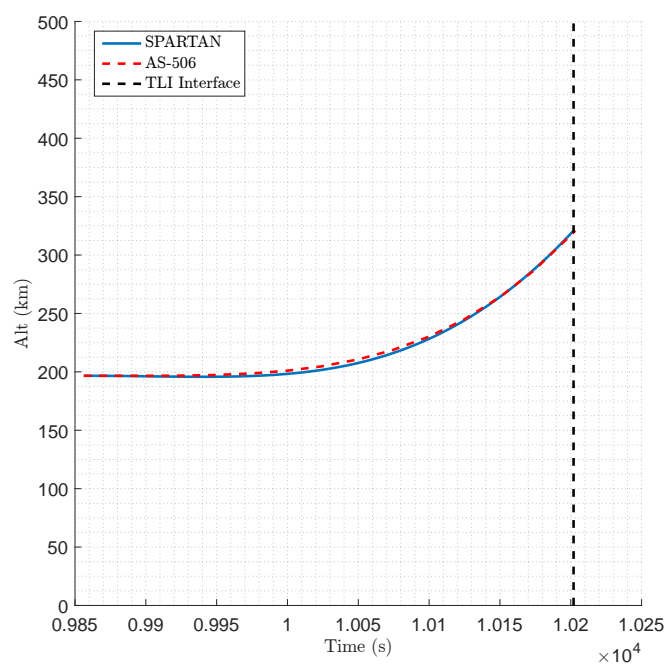

(a) Altitude profile

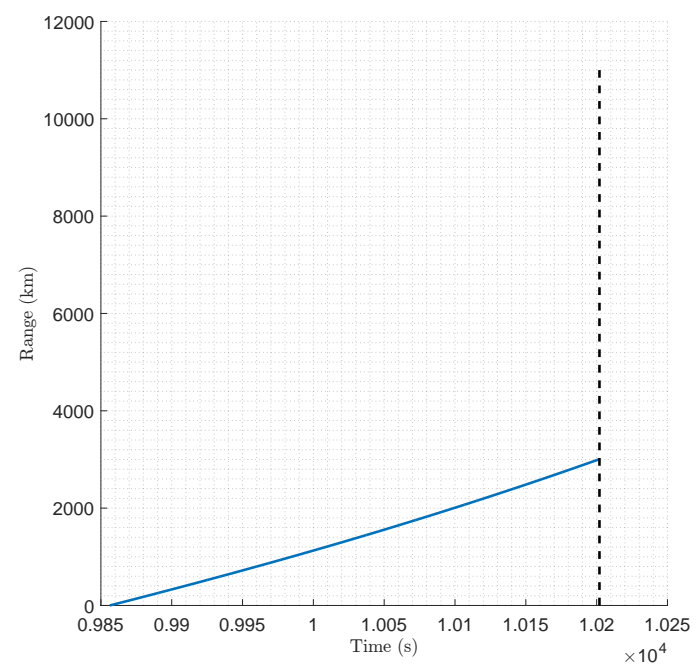

(b) Range profile

Fig. 11 TLI maneuver: (a) Altitude, and (b) Range.

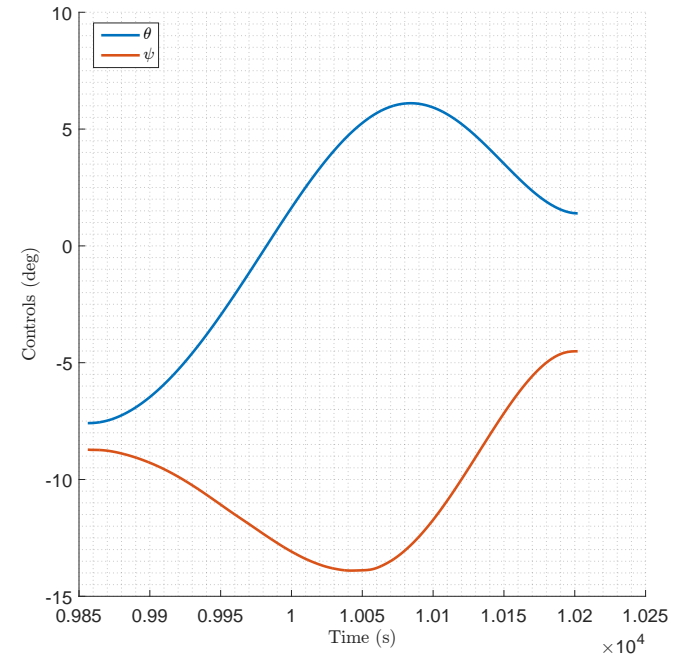

(a) Control profiles

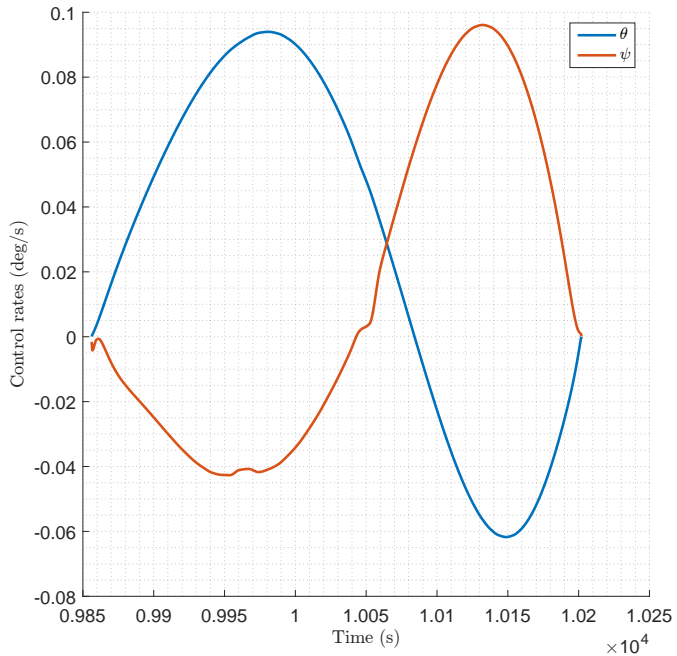

(b) Control rates profiles

Fig. 12 TLI maneuver: (a) Pitch and yaw, and (b) pitch and yaw rates. 


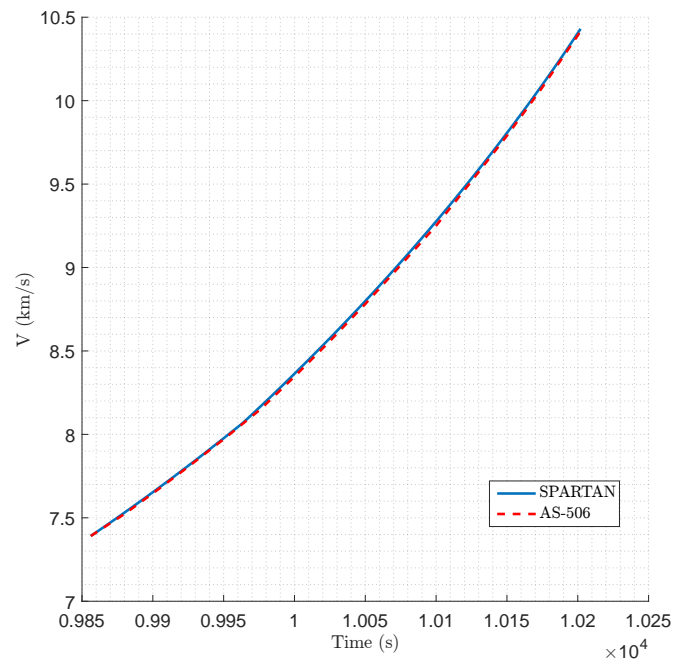

(a) Speed profile

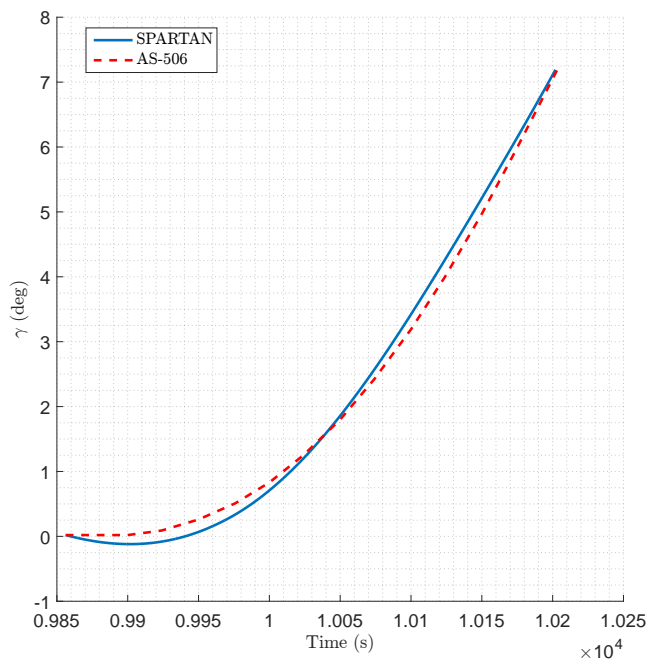

(b) Flight-path angle

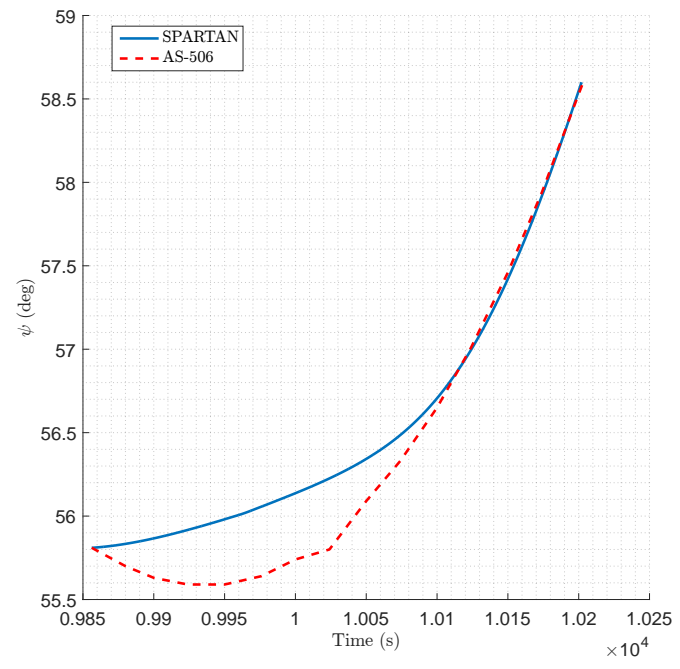

(c) Velocity azimuth angle

Fig. 13 TLI maneuver: (a) Speed, (b) Flight-path angle, and (c) Velocity-azimuth angle. 


\section{Moon Landing}

The most critical part of the mission was certainly the Moon landing. Once reached the sphere of influence of the Moon the spacecraft circularizes the orbit, and after a series of checks the Lunar Module (LM) (depicted in Fig. 14) separates from the Command and Service Module (CSM) to perform the Descent orbit insertion, an impulsive maneuver aiming at reducing the altitude of the LM to about $15 \mathrm{~km}$ (48000 ft) [32].

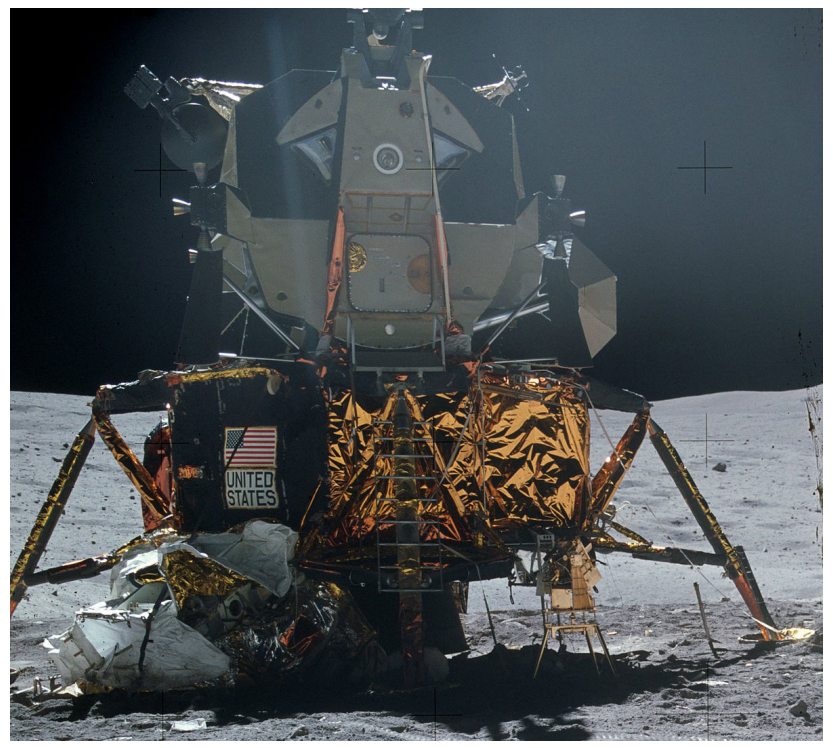

Fig. 14 Lunar Module, also known as LEM (Lunar Excursion Module), courtesy of NASA.

From here the powered descent maneuver begins. This maneuver is the subject of this section, and consists of three segments: the Braking phase, the Approach phase, and finally the Landing phase. As its name suggests the purpose of the Braking phase is to reduce the orbital speed of the LM by thrusting in opposite direction with respect to the motion. This phase started at a distance of about 260 nautical miles from the landing spot in the Sea of tranquility. It begins with the engine ignited and hold at about $10 \%$ of its maximum thrust during the first $26 \mathrm{~s}$ to be able to trim the spacecraft and ensure the correct attitude before throttling up to maximum thrust. In the last $120 \mathrm{~s}$ of this phase the thrust is reduced, and the engine becomes throttable, with throttle capability varying from 10 to $60 \%$ of its maximum value. This phase is concluded when the range-to-go is reduced to approximately 4.5 nautical miles from the landing site, after $506 \mathrm{~s}$. The on-board computer switches then to the approach phase.

The approach phase was meant to help the astronauts to monitor the situation while getting closer to the lunar surface through the frontal window of the LM. The attitude and the flight time are selected accordingly. The LM travels from about 4.5 nautical miles to about $2000 \mathrm{ft}$ from the landing spot during this phase, which takes in total $100 \mathrm{~s}$, and is considered terminated once that the altitude is decreased to $500 \mathrm{ft}$, where the landing phase begins.

When the landing sequence begins the forward velocity is about $60 \mathrm{ft} / \mathrm{s}$, with $16 \mathrm{ft} / \mathrm{s}$ of descent rate. The horizontal speed is progressively nullified until the point that the vehicle is able to vertically land.

The equations of motion used to describe the lunar landing dynamics are the following

$$
\left\{\begin{aligned}
\dot{\boldsymbol{r}} & =\boldsymbol{v} \\
\dot{\boldsymbol{v}} & =-\frac{\mu_{\circ}}{\|\boldsymbol{r}\|^{3}} \boldsymbol{r}+\frac{T}{m} \boldsymbol{u}-2 \boldsymbol{\Omega}_{\circ} \times \boldsymbol{v}-\boldsymbol{\Omega}_{\circ} \times \boldsymbol{\Omega}_{\circ} \times \boldsymbol{r} \\
\dot{m} & =-\frac{T}{g_{0} I_{s p}}
\end{aligned}\right.
$$

with the terms $\mu_{\circ}$ and $\Omega_{\circ}$ representing the Moon's gravitational parameter and rotation rate. Their values are assumed equal to $4.90486 \cdot 10^{12} \mathrm{~m}^{3} / \mathrm{s}^{2}$, and $2.6638 \cdot 10^{-6} \mathrm{rad} / \mathrm{s}$. The specific impulse $I_{s p}$ and the maximum thrust $T_{\max }$ are equal to $311 \mathrm{~s}$ and $45040 \mathrm{~N}$, respectively. Finally, the initial mass is $15103 \mathrm{~kg}$ [33]. These equations refer now to the Moon-Centered, Moon-Fixed (MCMF) reference frame. In this case since we have throttle capability at the end of 
the landing sequence we can rely not only on pitch and yaw as controls, but on the throttle level as well, that can vary between 0 (no thrust) and 1 (full throttle). For what concerns the cost function it is worth highlighting that we do not focus on the fuel-consumption optimality of the trajectory, given the reduced time-frame during which the throttleable capability is available. Instead, we focus again on the minimization of the control effort to come up with a smooth solution, and therefore we can rely again on Eq. (1) as function to be minimized.

Initial and final conditions were reconstructed by comparing different sources [28, 30, 32, 33] to come up with the following set of boundary conditions.

$$
\begin{aligned}
& \boldsymbol{r}\left(t_{0}\right)=\left[\begin{array}{lll}
1341880 & 1125971 & 31187
\end{array}\right]^{\top} \mathrm{m} \\
& v\left(t_{0}\right)=\left[\begin{array}{lll}
1075 & -1275 & -330
\end{array}\right]^{\top} \mathrm{m} \\
& m\left(t_{0}\right)=15103 \mathrm{~kg}
\end{aligned}
$$

and

$$
\begin{aligned}
\boldsymbol{r}\left(t_{F}\right) & =\left[\begin{array}{lll}
1591110 & 696710 & 22174
\end{array}\right]^{\top} \mathrm{m} \\
\boldsymbol{v}\left(t_{F}\right) & =\left[\begin{array}{lll}
-0.83 & -0.36 & -0.01
\end{array}\right]^{\top} \mathrm{m} \\
m\left(t_{F}\right) & =6903 \mathrm{~kg}
\end{aligned}
$$

To model this problem we chose to use 4 different phases: note that given the peculiarity of the original thrust profile the phases included in the optimization process do not temporarily match the sequence described at the beginning of the section, but are mainly divided according to the constraints acting on the thrust profile. In the first phase, lasting $26 \mathrm{~s}$, the throttle is constant and kept at $10 \%$. The second phase models the full-throttle segment of the braking phase, with throttle constant and equal to $100 \%$. A third phase with throttle fixed to $60 \%$ is then added to model the time ranging from 384 to $506 \mathrm{~s}$, to be consistent with the original profile depicted in [32], in Fig. 8 (a). Finally, the fourth phase is added to provide throttle control in the last $208 \mathrm{~s}$, and leads to the landing conditions on the surface of the Moon. Results are shown in Figs. 15-19.

In Fig. 15 the in-plane trajectory is depicted. The trajectory begins at about $500 \mathrm{~km}$ from the landing site. Note that since no accurate and unique longitude and latitude are available in the referenced sources the corresponding initial conditions in terms of positions are an estimate. The altitude is constantly reduced while the spacecraft approaches the landing site. A breakdown view of how the altitude decreases over the range flown by the LEM can be seen in Fig. 16. In the last phase the altitude is reduced down to $12 \mathrm{ft}$, when the touchdown sensors could detect the contact with ground and the engine cut-off can be issued. The throttle sequence is depicted in Fig. 17. Note that in the first three phases of optimization the algorithm correctly constrain the throttle to the prescribed levels (10\%, 100\%, and 60\%) before becoming free to change during the last phase. For what regards the attitude of the vehicle the pitch angle correctly decreases from the initial value of $90 \mathrm{deg}$ (to perform the braking maneuver to a value of 0 deg for the final touchdown, while the azimuth corrects for the initial side-motion. Whereas the results are overall satisfying, the resulting off-plane dynamics dominated by the azimuth angle is larger than the profile retrieved in the reference source data. Note that it was not possible to find the exact initial latitude and longitude at the moment of starting the powered descent sequence. Therefore an educated guess needed to be adopted, and together with some modeling mismatches it might be the reason explaining the difference in the yaw angle history. The rates, shown in Fig. 18(b), are satisfied as well. Finally, the plot showing the altitude vs the altitude rate is depicted in Fig. 19. This profile is sufficiently consistent with the original results of [28], Fig. 5-3, having a maximum difference of $15 \mathrm{~m} / \mathrm{s}$ in the middle of the descent that decreases to $0 \mathrm{~m} / \mathrm{s}$ towards the end of the mission profile, confirming the validity of the reconstruction. 


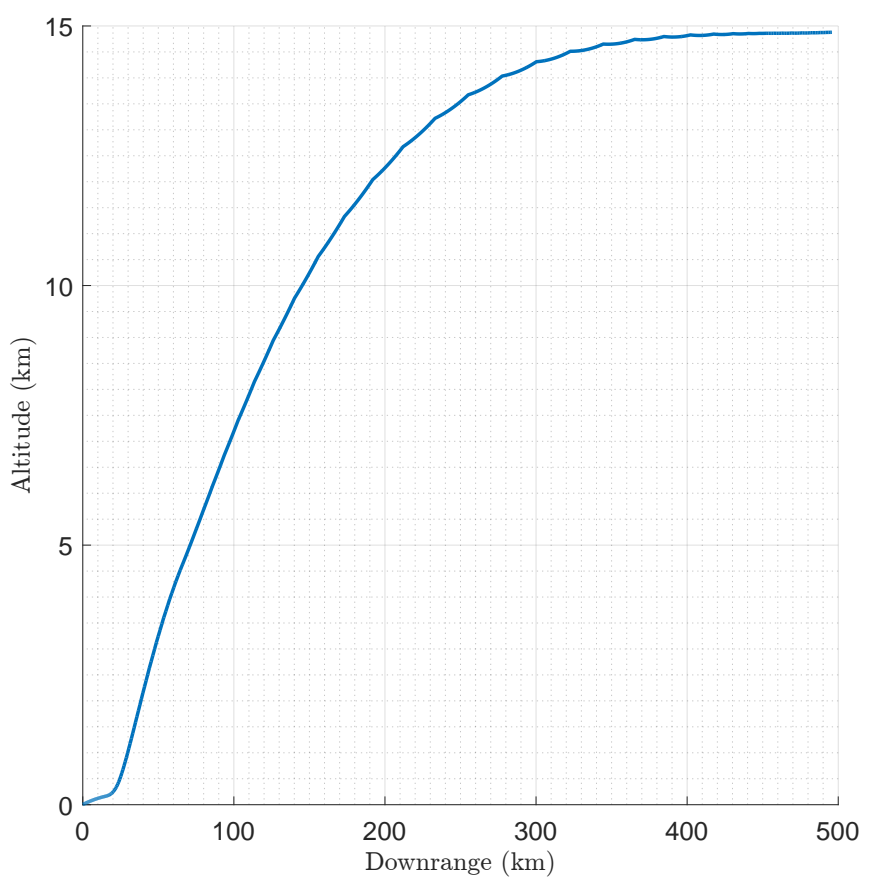

Fig. 15 Moon landing: Descent trajectory profile.
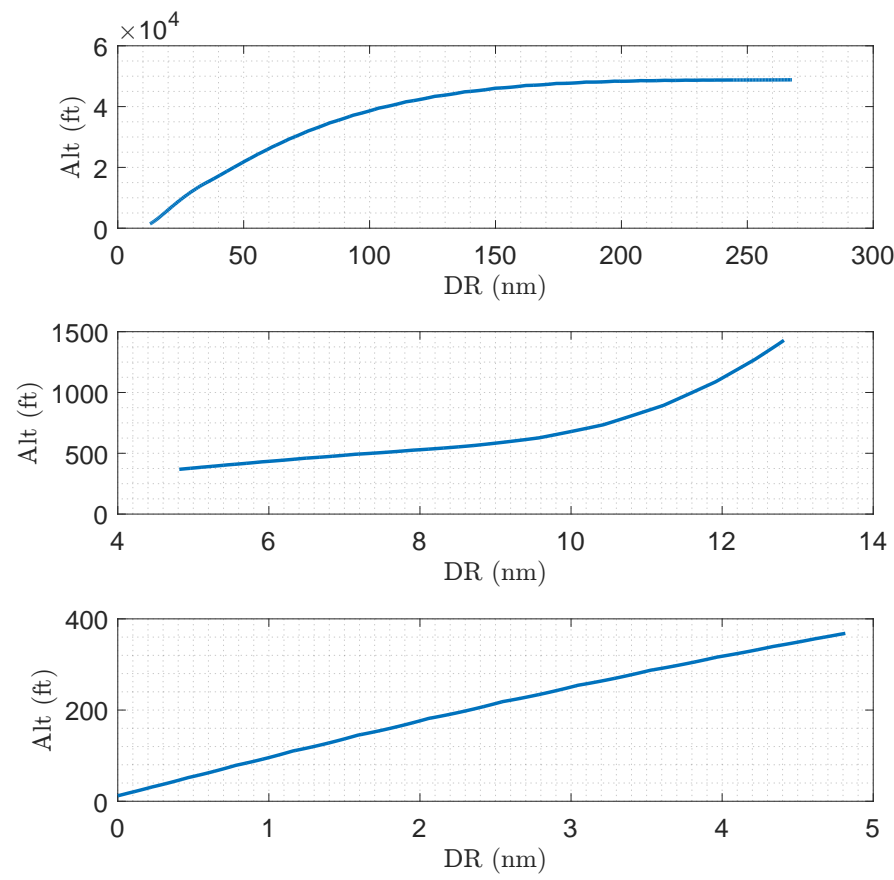

Fig. 16 Moon landing: Breakdown overview of altitude profile. 


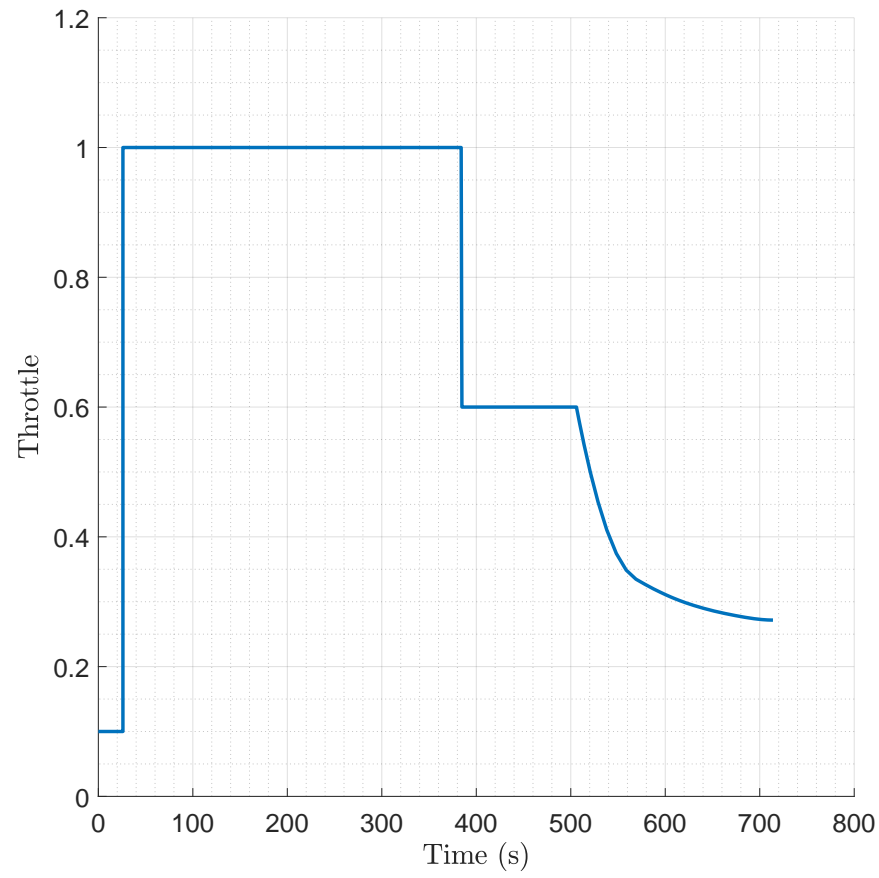

Fig. 17 Moon landing: Throttle profile.

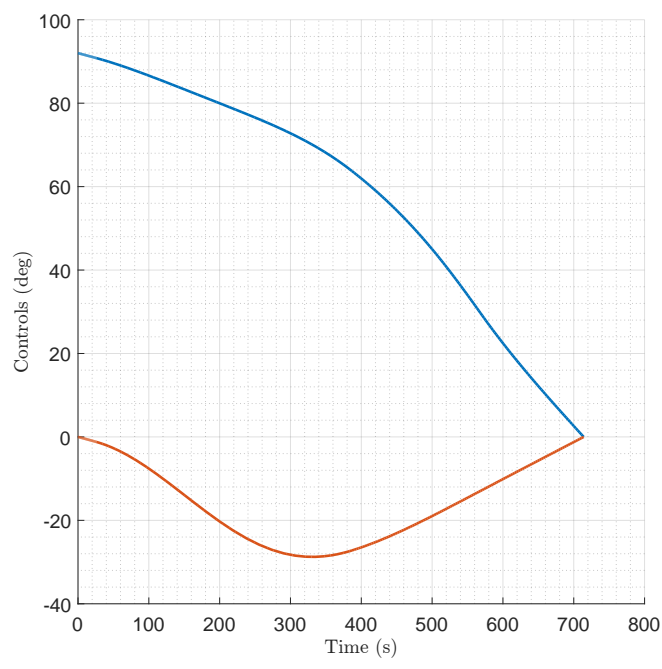

(a) Control profiles

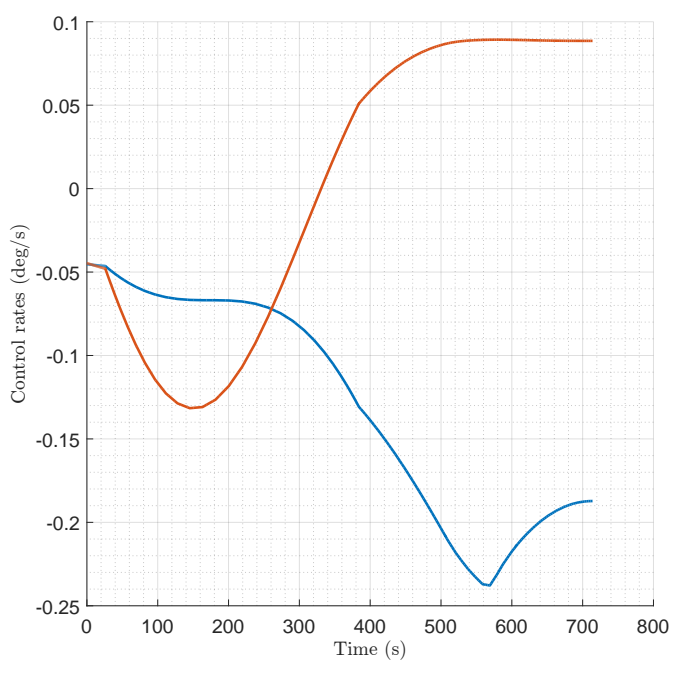

(b) Control rates profiles

Fig. 18 Moon Landing: (a) Pitch and yaw, and (b) pitch and yaw rates. 


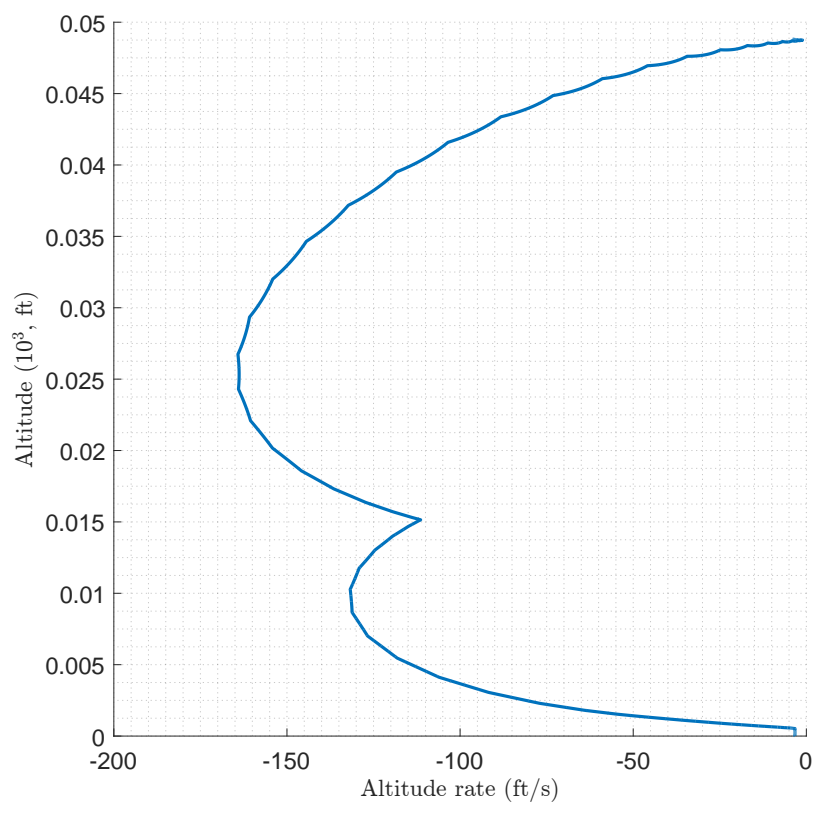

Fig. 19 Moon landing: Altitude vs altitude rate. 


\section{Conclusions}

This paper wants to be a tribute to the Apollo program and specifically to the Apollo 11 mission to celebrate the recent $50^{\text {th }}$ anniversary of one of the most important result achieved by the humankind in its persistent attempt to explore and understand the reality around us.

Although the efforts, the know-how and the passion of the original heroes that made this step possible remain unmatchable the technological progress over the last 50 years in terms of computational power and optimization algorithms allows nowadays to reconstruct within a modern framework the results that were the foundation of the Apollo program.

Some of these algorithms, and specifically pseudospectral methods, were used here to reconstruct critical phases of the Apollo 11 mission. These phases include the Saturn- $\mathrm{V}$ ascent, the translunar injection maneuver, and the Moon landing.

The computed solutions are, in the limits of some required simplifications, comparable to the original data available in the technical documentation released by NASA in 1969, confirming the validity of the proposed optimization-based reconstruction not only as design tool, but also as post-flight analysis methodology, here applied to an eminent test-case. Moreover, the proposed type of analysis could serve as framework to explore non-intuitive solutions, typically not visible with traditional design methods.

Future extensions of this work will include the reconstruction of the Moon ascent trajectory to perform the rendezvous with the Apollo Command and Service module, and the atmospheric entry of the Apollo 11 capsule that occurred on July, 24, 1969.

\section{References}

[1] Burns, K., and Turchak, L., "Sputnik - Why the Russians were First in Space," AIAA SPACE 2007 Conference \& Exposition, American Institute of Aeronautics and Astronautics, 2007. doi: 10.2514/6.2007-6063.

[2] Launius, R., "An Unintended Consequence of the IGY: Eisenhower, Sputnik, and the Founding of NASA," 46th AIAA Aerospace Sciences Meeting and Exhibit, American Institute of Aeronautics and Astronautics, 2008. doi: 10.2514/6.2008-860.

[3] Hyland, D. J., "Nationalism in Space Rhetoric, Khrushchev v. Kennedy and Burke - Looking to the Past to Ensure a More Cooperative Future," AIAA SPACE 2015 Conference and Exposition, American Institute of Aeronautics and Astronautics, 2015. doi: $10.2514 / 6.2015-4615$.

[4] Cadbury, D., Space Race: The Epic Battle Between America and the Soviet Union for Dominion of Space, Harper Perennial, 2007.

[5] Chertok, B., Rockets and People Vol IV: The Moon Race, CreateSpace Independent Publishing Platform, 2013.

[6] Launius, R. D., Apollo's Legacy: Perspectives on the Moon Landings, Smithsonian Books, 2019.

[7] O’Brien, F., The Apollo Guidance Computer, Springer, 2010.

[8] Haeussermann, W., "Description and Performance of the Saturn Launch Vehicle's Navigation, Guidance, and Control System," IFAC Proceedings Volumes, Vol. 3, No. 1, 1970, pp. 275-312. doi: 10.1016/s1474-6670(17)68785-8.

[9] Bryson Jr., A. E., and Ho, Y.-C., Applied Optimal Control. Optimization, Estimation, and Control, Hemisphere Publishing Corporation, 1975.

[10] MARTIN, D. T., and SIEVERS, R. F., "An empirical simulation of the translunar injection burn for Apollo." Journal of Spacecraft and Rockets, Vol. 6, No. 4, 1969, pp. 483-486. doi: 10.2514/3.29686.

[11] Klumpp, A. R., “Apollo lunar descent guidance,” Automatica, Vol. 10, No. 2, 1974, pp. 133-146. doi: 10.1016/00051098(74)90019-3.

[12] Lu, P., "Theory of Fractional-Polynomial Powered Descent Guidance," Journal of Guidance, Control, and Dynamics, Vol. 43, No. 3, 2020, pp. 398-409. doi: 10.2514/1.g004556.

[13] Nocedal, J., and Wright, S. J., Numerical Optimization, Springer-Verlag, New York, 1999.

[14] Betts, J. T., Practical Methods for Optimal Control and Estimation Using Nonlinear Programming, $2^{\text {nd }}$ ed., SIAM, Philadelphia, 2010. 
[15] Ross, I. M., Sekhavat, P., Fleming, A., Gong, Q., and Kang, W., "Pseudospectral Feedback Control: Foundations, Examples and Experimental Results," AIAA Guidance, Navigation, and Control Conference and Exhibit, American Institute of Aeronautics and Astronautics, 2006, pp. 1-23. doi: 10.2514/6.2006-6354.

[16] Ross, I. M., and Karpenko, M., "A review of pseudospectral optimal control: From theory to flight," Annual Reviews in Control, Vol. 36, No. 2, 2012, pp. 182-197. doi: 10.1016/j.arcontrol.2012.09.002.

[17] N. S. Bedrossian and S. Bhatt and W. Kang and I. M. Ross, "Zero-propellant maneuver guidance," IEEE Control Systems Magazine, Vol. 29, No. 5, 2009, pp. 53-73. doi: 10.1109/MCS.2009.934089.

[18] Bollino, K. P., Ross, I. M., and Doman, D., “Optimal Nonlinear Feedback Guidance for Reentry Vehicles,” AIAA Guidance Navigation, and Control Conference and Exhibit, Keystone, USA, 2006. doi: 10.2514/6.2006-6074.

[19] Sagliano, M., and Theil, S., "Hybrid Jacobian Computation for Fast Optimal Trajectories Generation," AIAA Guidance, Navigation, and Control Conference, Boston, USA, 2013. doi: 10.2514/6.2013-4554.

[20] Sagliano, M., Theil, S., Bergsma, M., D’Onofrio, V., Whittle, L., and Viavattene, G., "On the Radau Pseudospectral Method: theoretical and implementation advances," CEAS Space Journal, Vol. 9, No. 3, 2017, pp. 313-331. doi: 10.1007/s12567-0170165-5.

[21] Arslantas, Y. E., Oehlschlägel, T., and Sagliano, M., "Safe landing area determination for a Moon lander by reachability analysis," Acta Astronautica, Vol. 128, 2016, pp. 607 - 615. doi: http://doi.org/10.1016/j.actaastro.2016.08.013, URL http://www.sciencedirect.com/science/article/pii/S0094576516307846.

[22] Yan, H., Ross, I. M., and Alfriend, K. T., "Pseudospectral Feedback Control for Three-Axis Magnetic Attitude Stabilization in Elliptic Orbits," Journal of Guidance, Control, and Dynamics, Vol. 30, No. 4, 2007, pp. 1107-1115. doi: 10.2514/1.26591.

[23] Bittner, M., Fisch, F., and Holzapfel, F., "A Multi-Model Gauss Pseudospectral Optimization Method for Aircraft Trajectories," AIAA Atmospheric Flight Mechanics Conference, American Institute of Aeronautics and Astronautics, 2012. doi: 10.2514/6.20124728.

[24] Marco Sagliano and Stephan Theil and Vincenzo D’Onofrio and Michiel Bergsma, "SPARTAN: A Novel Pseudospectral Algorithm for Entry, Descent, and Landing Analysis," Advances in Aerospace Guidance, Navigation and Control, Springer International Publishing, 2017, pp. 669-688. doi: 10.1007/978-3-319-65283-2_36.

[25] Huneker, L., Sagliano, M., and Arslantas, Y., "SPARTAN: An Improved Global Pseudospectral Algorithm for High-Fidelity Entry-Descent-Landing Guidance Analysis," $30^{\text {th }}$ International Symposium on Space Technology and Science, Kobe, Japan, $2015,2015$.

[26] Garrido, J. V., and Sagliano, M., "Ascent and Descent Guidance of Multistage Rockets via Pseudospectral Methods," AIAA Scitech 2021, 2021. Submitted.

[27] Group, S. F. E. W., "Saturn V Launch Vehicle Flight Evaluation Report AS-506," Tech. Rep. NASA-TM-X-62558, NASA, 1969.

[28] Mission Evaluation Team, M. S. S., “Apollo 11 Mission Report,” Tech. Rep. NASA-MSC-00171, NASA, 1969.

[29] Braeunig, R. A., “Saturn V Launch Simulation,”, 2013. URL https://web.archive.org/web/20170313142729/http://www. braeunig.us/apollo/saturnV.htm, retrieval date 05-May-2020.

[30] Orloff, R., Apollo by the Numbers, US. Government Printing Office, 2001.

[31] NASA, “U.S. Standard Atmosphere 1976,” Tech. Rep. NASA Technical Memorandum X-74335, NASA, 1976.

[32] Bennett, F., "Lunar descent and ascent trajectories," Aerospace Sciences Meetings, American Institute of Aeronautics and Astronautics, 1970. doi: 10.2514/6.1970-25, URL http://dx.doi.org/10.2514/6.1970-25.

[33] NASA, “Apollo 11 Lunar Module / EASEP,”, 2015. URL Ahttps://nssdc.gsfc.nasa.gov/nmc/spacecraft/display.action?id=1969059C, retrieval date 20-May-2020. 